\title{
Dental caries diagnosis - toward the 21st century
}

To fill or not to fill? - a new technology may solve the dentist's dilemma.

For the majority of regular attenders, a visit to the dentist is no longer a painful proposition. In the century or so since Miller elaborated his "chemico-parasitic" theory of dental caries, there have been major advances in restorative techniques, new filling materials, and the use of implants to replace lost teeth. Lasers and air abrasion systems are being increasingly used to cut cavities, although they may never supplant the high-speed handpiece and dental bur. Despite these technical advances, dentistry, never the most popular of professions, has occasionally had to ride waves of negative publicity, such as generated by the amalgam controversy, the issue of infection control and sterilization, not to mention the vocal and still extremely active anti-fluoride lobby. In last month's issue of Nature Medicine, an article entitled "Detection of dental decay and its extent using a.c. impedance spectroscopy" by Longbottom et al. ${ }^{1}$, was published, which merits some comment. This paper describes exciting recent advances in technology that could be used in the future to aid the dental practitioner in the accurate detection and diagnosis of dental caries, but at the same time brings to light the disturbing fact that dentists are currently unable to accurately diagnose the disease they are supposedly treating. This fundamental failing is, to some extent, a reflection of the success of the profession in reducing rates of decay in the population.

As caries rates in the western world have declined to the point where $50 \%$ of 12 year-olds in the United States have not experienced the disease, there has been a concomitant alteration in the relative frequency of caries attack on different tooth surfaces; over $80 \%$ of new lesions now occur on the occlusal (biting) surfaces of the teeth. Lesions now take longer to develop, and remain at the early, or incipient, stage for a prolonged period. Dentistry's success in combating the disease has paradoxically amplified the problems of diagnosis, for the following reasons: (1) a higher proportion of lesions occur on tooth surfaces where diagnosis of the early lesion is problematic; (2) with a slower rate of progression, the clinician is more likely to encounter an early lesion; (3) given the same ability of diagnosis, a reduced prevalence of disease increases the number of false-positive decisions.

\section{Michael W.J. DoddS}

Caries is initially characterized by acid demineralization of the dental enamel, which occurs in a subsurface region before macroscopically visible cavitation, and is notoriously hard to diagnose. As the Iesion progresses into physical cavitation of the enamel, diagnosis becomes

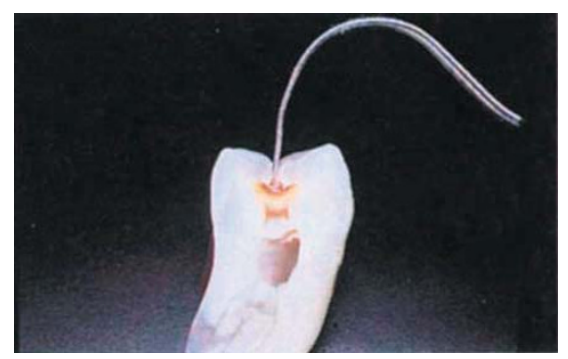

A new technique for detecting caries may allow dentists to discard their probes.

more straightforward. Since the acids are produced by bacterial fermentation of carbohydrate foodstuffs, lesions tend to be located in inaccessible sites where plaque accumulates undisturbed, usually in the occlusal fissures and approximal sites (where adjacent teeth abut).

Typically, caries is diagnosed by a combination of techniques, including visual inspection, radiographs and tactile sensation. The latter technique has been the mainstay of diagnosis for decades, and is characterized as a tug, or resistance to removal when the dental probe is inserted into an occlusal fissure (see figure). This technique has been criticized because of the possibility of transferring microorganisms from one site to another, as well as the risk of causing physical damage by insertion of a hard metal point into a site made physically fragile by demineralization. Finally, studies have indicated that the diagnostic power of the probe is low enough to warrant its removal from the diagnostic armamentarium. Radiographs can also be problematic in early caries diagnosis; an $\mathrm{X}$-ray is a two-dimensional picture of a three-dimensional structure, and since the enamel is highly radiopaque, a small amount of demineralization at one site may be masked by the radiodensity of the surrounding sound enamel. Improvements in the radiographic detection of caries lesions by computer digitiza- tion and enhancement techniques have been reported, but these techniques continue to be limited by the quality of the initial radiographic image. Every decision carries the risk of a false-positive or falsenegative diagnosis. False-positives will result in overtreatment and false-negatives in undertreatment or nontreatment of disease. A recent study ${ }^{2}$ compared methods of diagnosis for early (noncavitated) lesions and found that the combination of visual inspection and radiographs gave the best results, with sensitivity and specificity values of $49 \%$ and $87 \%$, respectively. Put into perspective, this means that half of the teeth with early caries went undiagnosed and, arguably of greater concern to many, $13 \%$ of teeth diagnosed with caries, and presumably thought to be in need of restoration, were in fact caries-free.

Following earlier studies that indicated that electrical resistance measurements of teeth held promise as a diagnostic technology, Longbottom et al. describe a more sophisticated method that collects complex impedance data and ultimately derives a single value for total impedance of the tooth. This method is able to distinguish between teeth with different severities of lesions, with impedance values from teeth in the different categories differing by a full order of magnitude, resulting in sensitivity and specificity values of $100 \%$. Not only does this far exceed any existing techniques of detection, but it should be independent of the experience and skill of the operator, who at present makes diagnostic judgement calls on a daily basis. A new technology that is able to accurately and validly diagnose and differentiate teeth based on caries severity would mean that a significant clinical problem would be resolved. Hopefully in the future, fewer teeth will be filled unnecessarily, and fewer cavities will go undetected. Dentists and patients alike should welcome this advance.

Department of Community Dentistry UT Health Science Center at San Antonio San Antonio, Texas 78284-7917, USA

1. Longbottorn, C., Huysmans, M-C.D.N.J., Pitts, N.B., Los, P. \& Bruce, P.G. Detection of dental decay and its extent using a.c. impedance spectroscopy. Nature Med. 2, 235-237 (1996).

2. Lussi, A. Comparison of different methods for the diagnosis of fissure caries without cavitation. Caries Res. 27, 409-416 (1993). 